\title{
Qualidade de vida em idosos que praticam atividade física - uma revisão sistemática
}

\author{
Quality of life in elderly people who practice physical activity - A systematic review
}

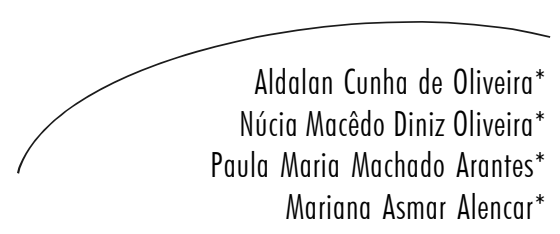

Objetivo: Realizar uma revisão sistematizada da literatura sobre o impacto da prática regular de atividade física na qualidade de vida dos idosos. Método: Revisão sistemática de estudos publicados até setembro de 2008, nas bases de dados Medline, LILACS e SciELO. Foram excluídos os estudos que possuíam delineamento transversal ou que eram relatos de casos, que avaliavam a qualidade de vida associada a alguma doença específica e aqueles nos quais havia presença de outra intervenção concomitante. Resultados: De acordo com os critérios de inclusão, dos 391 artigos encontrados, apenas três fizeram parte da análise. Foram identificados três tipos de intervenções: 1) exercícios de aquecimento, treino aeróbico, fortalecimento muscular, coordenação e resfriamento; 2) exercícios de fortalecimento muscular, treino funcional; 3) exercícios na água. Os resultados desses estudos sugerem que a qualidade de vida aumenta com a prática de atividade física (intervenções 1 e 3), principalmente se for realizada duas vezes por semana ao invés de somente uma vez. Os benefícios do treino de resistência muscular na qualidade de vida foram limitados e não foi encontrado benefício do treinamento funcional neste desfecho. Conclusão: $O$ número limitado de artigos encontrados, apenas um único estudo para cada tipo de intervenção, limita as conclusões desta revisão a respeito da eficácia dessas intervenções. Assim, conclui-se que há evidência limitada a respeito dos benefícios da prática de atividade física na qualidade de vida de idosos vivendo na comunidade. Sugere-se que mais estudos longitudinais sejam realizados para que as conclusões a respeito da eficácia destas intervenções sejam mais robustas.

\section{Abstract}

Objective: To conduct a systematic review of the literature regarding the impact of the practice of regular physical activity in the quality of life of elderly people. Methods: A systematic review of studies published until September 2008, in the databases Medline, LILACS and SciELO. It were excluded studies that had cross design or that were reports of cases that evaluated the quality of life associated with a specific disease and those in which there were concurrent presence of another intervention. Results: According to the inclusion criteria, of 391 articles found only three were part

\footnotetext{
Fundação Comunitária de Ensino Superior de Itabira. Curso de Fisioterapia.
}

\author{
Palavras-chave: Qualidade \\ de Vida. Exercício. Idoso. \\ Atividade Motora. \\ Treinamento de \\ Resistência. Atividade \\ Física.
}


of the analysis. We identified three types of interventions: 1) warming exercises, aerobic training, muscle strengthening, coordination and cooling, 2) muscle strengthening exercises, functional training, 3) exercises in water. The results of these studies suggest that the quality of life seems to increase with physical activity (interventions 1 and 3), especially if it is held twice a week instead of just once. The benefits of strength training for muscle in quality of life was limited and no benefit from functional training in this outcome was found. Conclusion: The limited number of articles found, only a single study for each type of intervention, limits the conclusions of this review regarding the effectiveness of these interventions. It is therefore concluded that there is limited evidence about the benefits of the practice of physical activity on the quality of life of elderly people living in the community. It is suggested that more longitudinal studies with different types of interventions be conducted so that the conclusion about the efficacy of these interventions will be more robust.
Key words: Quality of Life. Exercise. Elderly. Motor Activity. Resistance Training. Physical

\section{INTRODUÇÃO}

O envelhecimento populacional é um processo natural e manifesta-se por um declínio das funções de diversos órgãos que ocorre caracteristicamente em função do tempo, não se conseguindo definir um ponto exato de transição, como nas demais fases. ${ }^{1} \mathrm{O}$ envelhecimento é um processo que provoca alterações e desgastes em vários sistemas funcionais, que acontecem de forma progressiva e irreversível. O momento em que estas transformações surgem, quando passam a ser percebidas e como evoluem, diferencia-se de um indivíduo para o outro. ${ }^{2,3}$ Durante esse processo, diversas alterações afluem no organismo, como por exemplo, alterações fisiológicas, como o mau funcionamento de algumas estruturas corporais; e mecânicas, como a incapacidade de realizar movimentos básicos do corpo humano. ${ }^{4}$

No século XX, principalmente após a década de 50, houve uma mudança na pirâmide etária mundial. O processo de envelhecimento, que antes era restrito aos países desenvolvidos, está acontecendo nos países em desenvolvimento e de modo mais rápido. ${ }^{5}$ Dentre os países que apresentam maior ritmo de crescimento da população idosa, encontra-se o Brasil. A expectativa de vida dos brasileiros, que em 1900 não alcançava os 35 anos de idade, em 1950 atingiu 43 anos, em 2000, 68, com a expectativa de atingir os 80 anos em 2025..$^{6,7,8}$ Tal aumento colocará o Brasil, no ano de 2025, com a sexta população de idosos do mundo em termos absolutos. ${ }^{9} \mathrm{~A}$ tendência ao envelhecimento populacional está acarretando mudanças profundas em todos os setores da sociedade. ${ }^{10}$

O grande desafio que a longevidade coloca sobre os profissionais da área da saúde é o de conseguir uma sobrevida cada vez maior, com uma qualidade de vida cada vez melhor. Qualidade de vida $(Q V)$ se refere à percepção das pessoas de sua posição na vida, dentro do contexto de cultura e sistema de valores nos quais elas vivem e em relação a suas metas, expectativas e padrões sociais. ${ }^{11}$ Qualidade de vida é um conceito multidimensional que engloba critérios objetivos e mensuráveis, como funcionamento fisiológico ou a manutenção das atividades de vida diária, ${ }^{12}$ bem como componentes subjetivos, comumente designados por satisfação de vida, que traduzem o balanço entre as expectativas e os objetivos alcançados. ${ }^{13}$ Qualidade de vida é uma noção eminentemente humana, que tem sido aproximada ao grau de satisfação encontrado na vida familiar, amorosa, social e ambiental è própria estética existencial. O termo abrange muitos significados, que refletem conhecimentos, experiências e valores de indivíduos e coletividades que a ele se reportam em variadas épocas, espaços e histórias diferentes, sendo portanto uma construção social com a marca da relatividade cultural. Auquier et al. a qualificam como um conceito equívoco como o de inteligência, ambos dotados de um senso comum variável de um indivíduo ao outro. ${ }^{14}$ Martin $\&$ 
Stockler sugerem que qualidade de vida seja definida em termos da distância entre expectativas individuais e a realidade, sendo que, quanto menor a distância, melhor a qualidade de vida. ${ }^{15,16}$

Qualidade de vida na velhice tem sido definida como a percepção de bem-estar de uma pessoa, que deriva de sua avaliação do quanto realizou daquilo que idealiza como importante para uma boa vida e de seu grau de satisfação com o que foi possível concretizar até o momento. ${ }^{4}$ Além disso, à medida que um indivíduo envelhece, sua qualidade de vida é fortemente determinada por sua habilidade de manter autonomia e independência e, portanto, dependente do controle das prováveis doenças crônicas já presentes. ${ }^{4}$ Sendo assim, um envelhecimento bemsucedido é acompanhado de qualidade de vida e bem-estar e deve ser fomentado ao longo dos estados anteriores de desenvolvimento. ${ }^{17}$

Tem sido sugerido que os exercícios físicos podem ajudar as pessoas a manterem o maior vigor possível, melhorar a função em diversas atividades e assim, aumentar a qualidade de vida à medida que se envelhece. ${ }^{18} \mathrm{~A}$ prática de exercício físico, além de combater o sedentarismo, contribui de maneira significativa para a manutenção da aptidão física do idoso, seja na sua vertente da saúde, como nas capacidades funcionais. ${ }^{19}$ Outro benefício promovido pela prática de exercícios é a melhora das funções orgânicas e cognitivas, garantindo maior independência pessoal e prevenindo doenças. ${ }^{2}$

A atividade física é definida como qualquer movimento corporal produzido pelo músculo esquelético que resulta num aumento do dispêndio energético, constituindo-se processo complexo e dinâmico. ${ }^{20}$ Shephard enumera melhora da saúde, aumento da oportunidade de contatos sociais e ganhos na função cerebral como as principais razões para que indivíduos sedentários há anos, repentinamente, aos 70 ou 80 anos de idade, iniciem e mantenham a prática de exercícios físicos. ${ }^{21}$ As vantagens da prática de exercícios para idosos dependem de como se processa o envelhecimento e da rotina de exercício físico praticada. Sabe-se que os benefícios à saúde ocorrem mesmo quando a prática de atividade física é iniciada numa fase tardia de vida. ${ }^{5} \mathrm{Em}$ idosos, a atividade física regular é igualmente importante para o aumento ou preservação da força e da potência muscular, manutenção da mobilidade e da vida dependente, e prevenção e redução das quedas e das fraturas. ${ }^{22}$

Considerando todos os benefícios promovidos pela prática regular de atividade física, tem sido sugerido que esta seria uma alternativa para melhorar a qualidade de vida dos idosos. ${ }^{23,24} \mathrm{Lim}$ et al., em um estudo transversal, demonstraram que há uma correlação moderada e positiva entre a atividade física e os benefícios para a qualidade de vida. ${ }^{25}$ Uma vez que é importante não apenas aumentar os anos vividos pelos indivíduos, mas também aumentar concomitantemente a qualidade de vida durante esses anos, é importante que sejam realizados estudos que avaliem o impacto da prática de atividade física neste desfecho. Entretanto, não foi encontrado na literatura pesquisada nenhum estudo que fizesse uma revisão a respeito dos principais achados sobre os efeitos da prática de atividade física na qualidade de vida de idosos. Assim, este estudo teve como objetivo realizar uma revisão sistematizada sobre o impacto da prática regular de atividade física da população idosa na qualidade de vida desses indivíduos.

\section{METODOLOGIA}

\section{Estratégia de busca}

As bases de dados Medline, LILACS (Literatura Latino-Americana e do Caribe em Ciências da Saúde) e SciELO (Scientific Electronic Library Online) foram consultadas no período de agosto a setembro de 2008. Utilizou-se como limite de busca as palavras-chave constarem no título ou resumo do artigo e os idiomas dos artigos serem português, inglês ou espanhol. Foram usadas como palavras chave: quality of life; physical activity ou physical exercises e elderly or older or aged, com seus equivalentes em português (qualidade de vida, atividade física, exercícios físicos e idosos) e 
também em espanhol (calidad de vida, actividad fisica, ejercicios físicos, anciano). Durante a busca, houve o cruzamento dessas palavras.

\section{Seleção dos estudos}

Os títulos e os resumos escolhidos foram analisados por dois revisores independentes, que os classificaram como incluído, excluído ou duvidoso. Aqueles classificados como duvidosos foram revistos e discutidos por um terceiro revisor, para obtenção de um consenso. Foram considerados critérios de inclusão: 1) presença de grupo controle, com ou sem aleatorização, 2) participantes serem idosos, 3) utilizar a atividade física como intervenção, 4) apresentar a qualidade de vida como desfecho. Foram excluídos os estudos que possuíam delineamento transversal ou que eram relatos de casos, que avaliavam a qualidade de vida associada a alguma doença específica e aqueles nos quais havia presença de outra intervenção concomitante.

\section{Descrição dos estudos}

As principais informações dos estudos incluídos foram resumidas de forma padronizada. Estas informações foram organizadas nos tópicos: autor(es) e ano, características da amostra, desfechos avaliados e instrumentos utilizados para avaliação destes desfechos, características da intervenção (tipo de intervenção, frequência e duração das sessões, tempo total do tratamento) e os efeitos encontrados.

Foi utilizado um sistema de classificação por níveis de evidência para realizar a síntese dos resultados. A classificação inclui cinco categorias de evidência científica: evidência forte, limitada e moderada, achados indicativos e evidência insuficiente ou ausente (Anexo 1). ${ }^{26}$

\section{Avaliação da qualidade dos estudos}

A qualidade metodológica dos estudos foi avaliada utilizando-se a escala PEDro. Idealizada por Maher et al., essa escala é amplamente utilizada na área da reabilitação. Ela é composta de 11 itens, e a cada um dos itens presentes no estudo é somado um ponto (com exceção do item 1, que não é pontuado). Assim, o escore máximo é dez pontos e o mínimo, zero. A avaliação foi feita por dois autores de forma independente. Estudos prévios demonstraram níveis aceitáveis de confiabilidade entre examinadores. ${ }^{27}$ Nos casos de discordância, era consultado um terceiro avaliador.

\section{RESULTADOS}

A pesquisa inicial, realizada nas bases de dados eletrônicas, identificou 391 artigos. Após a análise dos títulos, 126 deles foram selecionados. Posteriormente, os resumos foram lidos e foram excluídos dez artigos, por terem delineamento transversal, três por serem relato de caso, 59 por não usarem a qualidade de vida como desfecho, 29 por apresentarem intervenção concomitante e 21 por terem na população avaliada a qualidade de vida associada a alguma doença específica. Foi excluído um artigo desta revisão por não estar disponível nas bases de dados e após solicitado, não chegaria em tempo hábil para ser incluído no estudo. Conforme os critérios de inclusão predeterminados, três artigos foram escolhidos para participarem da revisão sistemática. Ocorreu uma variabilidade em relação ao tipo de intervenção utilizada, percebendo-se três tipos diferentes de intervenção. $O$ desfecho analisado em todos os estudos foi a qualidade de vida. A maioria dos estudos utilizou o SF-36 como instrumento de medida, que investiga oito domínios da QV distribuídos em 36 questões sobre: função física, percepção de dor corporal, saúde geral, vitalidade, função social, limitações devido à saúde emocional, limitações devido à saúde física e saúde mental. ${ }^{28} \mathrm{Nem}$ todos os artigos utilizaram o SF-36.

Quanto às intervenções pesquisadas, os estudos demonstram que: a duração do programa de atividade física (semanas/meses) variou entre dez semanas e seis meses; a duração das sessões (horas/ minutos) entre 45 minutos a uma hora e a frequência (número de vezes por semana) de uma a três vezes por semana. As características dos artigos selecionados quanto à intervenção, aos desfechos e aos resultados são apresentadas no quadro 1. 
Quadro 1 - Características dos artigos selecionados nesta revisão sistemática.

\begin{tabular}{|c|c|c|c|c|}
\hline Autor/Ano & Participantes & Desfechos & Intervenção & $\begin{array}{l}\text { Efeitos encontrados (de acordo } \\
\text { com a numeração dos } \\
\text { desfechos) }\end{array}$ \\
\hline $\begin{array}{l}\text { Stiggelbout et } \\
\text { al., } 2004\end{array}$ & $\begin{array}{l}\mathrm{n}=277(\mathrm{GE}: \\
\text { MBV01 } \mathrm{n}=98 \mathrm{e} \\
\text { MBV02 } \mathrm{n}=53) \\
(\mathrm{GC} \mathrm{n}=126) \\
65 \text { a } 80 \text { anos, } \\
\text { independentes. }\end{array}$ & $\begin{array}{l}\text { Efeitos do } \\
\text { exercício sobre a } \\
\text { QV relaciona da } \\
\text { à saúde de idosos } \\
\text { independentes. } \\
\text { (Vitality Scale } \\
\text { Plus, TAAQOL } \\
\text { e RAND-36). }\end{array}$ & $\begin{array}{l}\text { Duração: } 10 \text { semanas. } \\
\text { MBV01 fazia 1x por semana e } \\
\text { MBV02 2x por semana. } \\
\text { MBV01 e MBV02 faziam } 45 \\
\text { min de exercícios sendo, } 5 \text { min } \\
\text { de aquecimento, } 35 \text { min de } \\
\text { aeróbico, fortalecimento, } \\
\text { exercício de coordenação e } 5 \\
\text { min de resfriamento. } \\
\text { GC participava de um } \\
\text { programa de educação } \\
\text { sanitária (interação social, } \\
\text { saúde, educação sobre estilo de } \\
\text { vida, beneficio da AF, } \\
\text { exercício e alimentação). } 1,5 \mathrm{~h} \\
\text { mensais. }\end{array}$ & $\begin{array}{l}\text { Após } 10 \text { semanas o MBV01 } \\
\text { não teve melhora na QV } \\
\text { quando comparado ao MBV02 } \\
\text { (vitalidade } \mathrm{p}=0,51 \text {; dor } \\
\mathrm{p}=0,38 ; \text { saúde mental } \mathrm{p}=0,40 ; \\
\text { sensação geral da saúde } \mathrm{p}=0,99 \\
\text { e mudança no estado da saúde } \\
\mathrm{p}=0,56 \text { ). MBV01 ao contrário } \\
\text { do MBV02 não apresentou } \\
\text { benefício em relação ao estado } \\
\text { funcional }\end{array}$ \\
\hline $\begin{array}{l}\text { Sato et al., } \\
2007\end{array}$ & $\begin{array}{l}\mathrm{N}=30 \mathrm{G} 1 \mathrm{n}=10 \\
\mathrm{G} 2 \mathrm{n}=12, \mathrm{GC} \\
\mathrm{n}=8 \\
\geq \text { que } 65 \text { anos, } \\
\text { sedentários com } \\
\text { baixo nível de } \\
\text { cuidados de } \\
\text { enfermagem. }\end{array}$ & $\begin{array}{l}\text { Efeitos do } \\
\text { exercício físico } \\
\text { na água sobre a } \\
\text { QV. (SF - } 36 \\
\text { antes, após } 3 \text { e } 6 \\
\text { meses) }\end{array}$ & $\begin{array}{l}\text { Duração: } 6 \text { meses. } \\
\text { G1 fazia } 1 \text { x por semana e G2 } \\
\text { fazia 2x por semana. G1 e G2 } \\
\text { participaram de um programa } \\
\text { de exercícios sendo } 10 \text { min de } \\
\text { aquecimento em terra e } 50 \\
\text { min na água ( } 20 \text { min de } \\
\text { caminhada, } 10 \text { min de } \\
\text { exercícios funcionais, } 10 \text { min } \\
\text { de alongamento e resistência e } \\
10 \text { min de relaxamento). } \\
\text { GC participava de atividades } \\
\text { sociais e de recreação (lazer, } \\
\text { comunicação). }\end{array}$ & $\begin{array}{l}\text { O componente físico e o } \\
\text { mental em } 3 \text { e } 6 \text { meses } \\
\text { aumentaram } \\
\text { significativamente em relação } \\
\text { ao pré em G1 e G2 ( } p<0,05 \text { ). } \\
\text { O componente físico em G2 } \\
\text { foi significativamente superior } \\
\text { ao GC em } 3 \text { meses ( } p<0,05 \text { ). } \\
\text { Aos } 6 \text { meses o compon ente } \\
\text { físico foi semelhante em G1 e } \\
\text { G2. G1 e G2 aos } 3 \text { meses } \\
\text { foram semelhantes no } \\
\text { componente mental. Após } 6 \\
\text { meses a limitação física ( } \\
p<0,000) \text {, dor corporal } \\
\text { ( } p=0,073 \text { ), saúde geral } \\
\text { ( } p=0,006 \text { ), vitalidade } \\
\text { ( } p<0,000 \text { ), função social } \\
\text { ( } p<0,000 \text { ) e limitação por } \\
\text { aspectos emocionais ( } p=0,003 \text { ) } \\
\text { melhoraram } \\
\text { independentemente da } \\
\text { freqüência do exercício. O } \\
\text { componente físico, limitação } \\
\text { física, saúde geral e limitação } \\
\text { por aspectos emocionais } \\
\text { tiveram diferença nos } G 1 \text { e } G 2 \\
\text { após } 3 \text { meses e mantiveram-se } \\
\text { invariáveis após } 6 \text { meses. No } \\
\text { GC houve diferença das } \\
\text { limitações físicas e vitalidade } \\
\text { em } 6 \text { meses e no } \\
\text { funcionamento físico em } 3 \\
\text { meses. }\end{array}$ \\
\hline
\end{tabular}


Quadro 1 - Características dos artigos selecionados nesta revisão sistemática. (continuação)

\begin{tabular}{|c|c|c|c|c|}
\hline Autor/Ano & Participantes & Desfechos & Intervenção & $\begin{array}{l}\text { Efeitos encontrados (de acordo } \\
\text { com a numeração dos } \\
\text { desfechos) }\end{array}$ \\
\hline $\begin{array}{l}\text { De Vreede et } \\
\text { al., } 2007\end{array}$ & $\begin{array}{l}\mathrm{n}=74 \mathrm{G} 1 \text { (grupo } \\
\text { funções): } \mathrm{n}=30 \\
\mathrm{G} 2 \text { (grupo } \\
\text { resistência) } \mathrm{n}=28 \\
\mathrm{GC} \mathrm{n}=26 \\
\text { Mulheres } \geq 70 \\
\text { anos, saudáveis. }\end{array}$ & $\begin{array}{l}\text { Efeitos dos } \\
\text { exercícios de } \\
\text { funcionamento } \\
\text { físico e de } \\
\text { resistência na } \\
\text { QV. (SF -36 } \\
\text { aplicado no } \\
\text { início, após a } \\
\text { intervenção aos } \\
3 \text { meses e } 6 \\
\text { meses mais } \\
\text { tarde). }\end{array}$ & $\begin{array}{l}\text { Duração: } 12 \text { semanas. } \\
\text { G1 e G2 faziam 3x por semana } \\
\text { por } 1 \mathrm{~h} \\
\text { Grupo função fazia exercícios } \\
\text { de treinamento funcional ( } 3 \\
\text { séries de } 5 \text { a } 10 \text { repetições) e } \\
\text { Grupo resistência fazia } \\
\text { treinamento de resistência } \\
\text { para fortalecimento muscular } \\
\text { ( } 3 \text { séries de } 10 \text { repetições). A } \\
\text { intensidade foi avaliada por } \\
\text { escala de auto-percepção de } \\
\text { esforço e graduada de } \\
\text { moderada a alta. } \\
\text { GC não realizou. }\end{array}$ & $\begin{array}{l}\text { Aos } 3 \text { meses não ocorreu } \\
\text { nenhuma diferença } \\
\text { significativa entre as mudanças } \\
\text { nas médias dos escores de QV } \\
\text { dos três grupos, exceto para o } \\
\text { domínio funcionamento físico. } \\
\text { Neste domínio, o aumento da } \\
\text { QV foi significativamente } \\
\text { maior no grupo resistência que } \\
\text { no grupo função (p=0,040) e } \\
\text { GC (p=0,019). Seis meses após } \\
\text { o encerramento da } \\
\text { intervenção, G1 e G2 } \\
\text { mostraram ter uma } \\
\text { diminuição do escore de QV. } \\
\text { Em } 9 \text { meses G2 teve uma } \\
\text { menor pontuação da QV do } \\
\text { que no início do estudo. } \\
\text { Comparando os escores aos } 9 \\
\text { meses em relação aos valo res } \\
\text { basais, o GC demonstrou uma } \\
\text { tendência de aumento nos } \\
\text { componentes físicos. Entre } 3 \text { e } \\
9 \text { meses, G1 apresentou uma } \\
\text { diminuição no escore do } \\
\text { domínio funcionamento físico } \\
\text { abaixo dos valores basais } \\
\text { (p=0,026). Entre } 3 \text { e } 9 \text { meses o } \\
\text { funcionamento físico e o } \\
\text { componente físico diminuíram } \\
\text { no GC em relação ao G1. }\end{array}$ \\
\hline
\end{tabular}

GE: grupo experimental; GC: grupo controle; AF: atividade física: QV: qualidade de vida.

Quanto à população total pesquisada, podese perceber o predomínio de idosos saudáveis que viviam na comunidade, do sexo feminino, da cor branca e sedentários. A presença de similaridade entre os grupos ou uma amostra homogênea dos grupos no pré-tratamento, foi encontrada na maioria dos artigos selecionados. ${ }^{28,29}$

1. Exercícios de aquecimento, treino aeróbico, fortalecimento muscular, coordenação e resfriamento

Foi encontrado apenas um estudo contemplando a avaliação dos efeitos deste tipo de intervenção em idosos saudáveis vivendo na comunidade. ${ }^{29}$ Este demonstrou que os exercícios realizados uma vez por semana não são suficientes para melhorar a qualidade de vida a curto prazo. No entanto, quando esses idosos realizaram exercícios duas vezes por semana, os aspectos vitalidade, percepção de dor corporal, saúde mental, percepção geral da saúde e limitações devido à saúde física avaliados no RAND-36 melhoraram significativamente. ${ }^{28}$ Isso demonstra que essa população adquiriu benefícios em relação à qualidade de vida.

\section{Exercícios de fortalecimento muscular e treino funcional}

Um estudo avaliou os efeitos de um programa de exercícios de fortalecimento muscular e um programa de treino de atividades funcionais na 
qualidade de vida de idosas saudáveis vivendo na comunidade. ${ }^{30}$ Esse estudo demonstrou que os valores da função física das idosas que realizaram exercícios de fortalecimento muscular aumentaram significativamente, quando comparadas ao grupo que realizou exercícios de treino funcional e ao grupo controle. Mas, em contrapartida, após nove meses, observou-se menor pontuação da qualidade de vida nas idosas que realizavam os dois tipos de exercícios. Portanto, concluíram que o exercício físico, praticado na forma de exercícios com resistência para fortalecimento muscular ou treino funcional, tem efeito limitado sobre a qualidade de vida nas mulheres idosas.

\section{Exercícios na água}

Apenas um estudo avaliou o efeito do exercício realizado na água na qualidade de vida em idosos saudáveis. ${ }^{28} \mathrm{O}$ estudo investigou o efeito $\mathrm{da}$ intervenção uma vez por semana e duas vezes por semana num período de seis meses. Após três meses de intervenção, verificou-se aumento significativo para os componentes físico e mental do questionário SF-36, comparando com a avaliação pré-intervenção. Entretanto, em relação ao componente físico, este aumento foi significativamente maior no grupo que realizava os exercícios $2 \mathrm{x}$ por semana em relação ao grupo que realizava $1 \mathrm{x}$ por semana e apenas o grupo que realizava os exercícios $2 \mathrm{x}$ por semana apresentou diferença em relação ao grupo controle. Após seis meses, não houve diferença entre o grupo que fazia $1 \mathrm{x}$ por semana $\mathrm{e} 2 \mathrm{x}$ por semana neste componente. Não foram verificadas diferenças no grupo controle. Após seis meses, os aspectos limitação física, percepção de dor corporal, saúde geral, vitalidade, função social e limitação por aspectos emocionais tiveram seus escores mais elevados independentemente da frequência do exercício.

Em relação à qualidade dos estudos, dois $\operatorname{artigos}^{29,30}$ apresentaram nota 5 na escala PEDro e um estudo ${ }^{28}$ obteve seis pontos nesta escala. As pontuações dos artigos em cada critério estão descritas no quadro 2 .

Quadro 2 - Classificação metodológica avaliada pela escala PEDro (2003).

\begin{tabular}{|lccc|}
\hline & Sato (2007) & $\begin{array}{c}\text { De Vreede } \\
(2007)\end{array}$ & $\begin{array}{c}\text { Stiggelbout } \\
(2005)\end{array}$ \\
\hline 1- Especificação de critérios de inclusão (item não pontuado) & $\mathrm{S}$ & $\mathrm{S}$ & $\mathrm{S}$ \\
\hline 2- Alocação aleatória & $\mathrm{S}$ & $\mathrm{S}$ & $\mathrm{S}$ \\
\hline 3- Sigilo na alocação & $\mathrm{S}$ & $\mathrm{N}$ & $\mathrm{N}$ \\
\hline 4- Similaridade inicial dos grupos & $\mathrm{S}$ & $\mathrm{S}$ & $\mathrm{S}$ \\
\hline 5-Mascaramento de participantes & $\mathrm{N}$ & $\mathrm{N}$ & $\mathrm{N}$ \\
\hline 6- Mascaramento do terapeuta & $\mathrm{N}$ & $\mathrm{N}$ & $\mathrm{N}$ \\
\hline 7- Mascaramento do avaliador & $\mathrm{N}$ & $\mathrm{N}$ & $\mathrm{S}$ \\
\hline 8- Medidas de um desfecho primário (85\% dos participantes) & $\mathrm{S}$ & $\mathrm{S}$ & $\mathrm{N}$ \\
\hline 9- Análise de intenção de tratar & $\mathrm{N}$ & $\mathrm{N}$ & $\mathrm{N}$ \\
\hline 10- Comparação entre grupos em um desfecho primário & $\mathrm{S}$ & $\mathrm{S}$ & $\mathrm{S}$ \\
\hline $\begin{array}{l}\text { 11-Tendência central e variabilidade de pelo menos uma } \\
\text { variável }\end{array}$ & $\mathrm{S}$ & $\mathrm{S}$ & $\mathrm{S}$ \\
\hline Total (em 10 pontos) & 6 & 5 & 5 \\
\hline
\end{tabular}

Legenda: S- sim; N- Não 


\section{DISCUSSÃO}

Apesar de a qualidade de vida ter-se tornado um dos mais importantes temas contemporâneos, esta revisão sistemática mostra um número limitado de ensaios clínicos relacionados com a prática de atividade física e seus efeitos na qualidade de vida de idosos vivendo na comunidade. A maioria dos estudos encontrados apresentava delineamento transversal. Este tipo de delineamento não permite avaliar a eficácia da intervenção, uma vez que é realizada apenas uma avaliação dos idosos e não há um acompanhamento destes idosos antes e após a realização de um programa de atividade física. Assim, estes estudos não foram incluídos nesta revisão, uma vez que eles não permitem estabelecer uma relação causal entre a prática de atividade física e o desfecho qualidade de vida.

Apesar de todos os estudos incluídos nesta revisão utilizarem como intervenção a atividade física, os três artigos apresentaram diferenças nas formas de intervenção. Todos eles utilizaram exercícios a serem realizados em ambiente clínico, mas diferiram na forma como eles eram executados. Um estudo usou a água para a prática das atividades físicas, ${ }^{28}$ o outro utilizou exercícios combinados (treino aeróbico, fortalecimento e exercícios de coordenação) ${ }^{29} \mathrm{e}$ um terceiro utilizou exercícios específicos (treinamento funcional e treinamento de resistência). ${ }^{30}$

Em relação à prática de atividade física na água e ao programa composto por exercícios aeróbicos, de fortalecimento e de coordenação, houve evidência limitada sobre a eficácia dessas intervenções na qualidade de vida. Além disto, os dois estudos demonstraram que os efeitos dessas intervenções foram melhores quando a atividade era realizada duas vezes por semana. ${ }^{28,29}$ Sato et al. demonstraram que, após três meses de exercícios na água, a melhora no componente físico da qualidade de vida foi maior quando se praticava essa atividade duas vezes por semana, se comparado a uma vez por semana. Aos seis meses, esse componente foi similar nos respectivos grupos. ${ }^{28}$ Resultado similar foi encontrado por
Stiggelbout et al..$^{29}$ Ao avaliar a qualidade de vida em participantes de atividades físicas uma e duas vezes por semana, durante dez semanas, os autores concluíram que o grupo que praticava as atividades (exercício aeróbico, de fortalecimento e coordenação) uma vez por semana não apresentou melhora na qualidade de vida. Já o grupo que praticava as mesmas atividades duas vezes por semana apresentou melhora significativa. ${ }^{29}$ Apesar de a amostra, a duração da intervenção e o tempo de realização das atividades nos dois estudos deferirem muito entre si, podese perceber que, nos dois estudos, os grupos que praticavam essas atividades duas vezes por semana apresentaram melhora na qualidade de vida superior a dos grupos que praticavam apenas uma vez na semana. Assim, parece que a frequência com a qual o idoso pratica a atividade influencia na melhora da qualidade de vida.

Como descrito anteriormente, um estudo avaliou os efeitos dos exercícios de treinamento funcional e treinamento de resistência na qualidade de vida de idosas saudáveis vivendo na comunidade..$^{30}$ Os dois grupos realizavam estes exercícios três vezes por semana, com duração de doze semanas. Aos três meses, houve aumento apenas no domínio funcionamento físico no grupo de resistência. Aos seis meses, após a intervenção, os dois grupos mostraram ter uma diminuição do escore de QV, evidenciando que o benefício na QV não se manteve após o término do exercício. Além disso, um terceiro grupo (o grupo controle), que não realizou nenhuma intervenção, nesse mesmo momento demonstrou uma tendência de aumento nos componentes físicos. Assim, este estudo sugere que o treino funcional tem efeito limitado sobre a QV de mulheres idosas e que a realização de exercícios de resistência não combinados a outros tipos de exercícios não tem efeitos sobre a qualidade de vida de idosas. A classificação por níveis de evidência indica a incontestável insuficiência a respeito da eficácia dessas intervenções na qualidade de vida.

Estudos transversais têm demonstrado que idosos que praticam exercícios físicos apresentam 
melhor qualidade de vida do que os idosos que não praticam. ${ }^{23,24,25}$ Mota et al. demonstraram que idosos que participaram de um programa de atividade física (caminhada, fortalecimento muscular, resistência, flexibilidade e equilíbrio), realizado duas vezes por semana e durante dez meses, apresentavam melhor qualidade de vida após o término deste programa que os idosos que não participaram deste. ${ }^{24}$ Yasunaga et al. ${ }^{31}$ avaliaram se o aumento da quantidade de atividade física traz benefícios para qualidade de vida em idosos saudáveis vivendo na comunidade. Para isso, usaram um acelerômetro eletrônico para contar os passos dos idosos 24 horas por dia, durante um ano. Os autores encontraram uma associação entre o volume de atividade física e a qualidade de vida global. ${ }^{31}$

Apesar de a caminhada ser uma atividade realizada com muita frequência pelos idosos, não foi encontrado nenhum estudo que avaliasse os efeitos dessa atividade na qualidade de vida dos idosos. Estudo transversal conduzido por Brown et al. concluiu que os idosos que relatam praticar caminhada leve, corrida e ciclismo regularmente estão mais propensos à melhora da qualidade de vida global do que os que relatam não praticar nenhuma atividade física. ${ }^{23}$ Entretanto, pelo fato de o delineamento do estudo ser transversal, não é possível concluir sobre a eficácia dessa atividade na qualidade de vida, sendo necessária a realização de ensaios clínicos para investigar esta questão.

A qualidade metodológica dos estudos foi averiguada nesta revisão. Os três estudos aqui incluídos apresentaram alocação aleatória. Em relação ao mascaramento dos avaliadores, apenas o estudo de Stiggelbout et al. mascarou o avaliador, o que é especialmente importante em estudos que avaliam a qualidade de vida, pois a expectativa do examinador em relação à melhora deste desfecho pode influenciar o desempenho do participante. ${ }^{29}$

A partir da leitura e análise dos artigos que integraram esta revisão, pode-se perceber que os valores da qualidade de vida em idosos parecem aumentar quando participam de alguma atividade física como exercício na água e o treino aeróbico associado à coordenação e fortalecimento. Apesar de os estudos divergirem quanto ao tempo de duração, a amostra estudada, o tipo de intervenção e ao modo como avaliaram a QV, esses estudos apontaram para esta conclusão. Também se observou que a prática desses exercícios com uma frequência de duas vezes por semana parece ser mais benéfica para a qualidade de vida que a prática com apenas uma vez por semana. ${ }^{28,29}$ Em relação ao treinamento funcional e treinamento de resistência, praticados de forma isolada, parece haver um efeito limitado e não duradouro dessas intervenções na qualidade de vida. ${ }^{30}$

O número restrito de artigos encontrados, apenas um único estudo para cada tipo de intervenção, limita as conclusões desta revisão a respeito da eficácia dessas intervenções. Os resultados de apenas um ensaio clínico não são suficientes para esclarecer sobre a eficácia de uma determinada intervenção. ${ }^{32}$ As conclusões são mais robustas quando diferentes estudos investigam os efeitos de uma intervenção e fornecem dados que suportam as mesmas conclusões. ${ }^{32}$ Assim, é necessário que mais ensaios clínicos que avaliem o impacto das diversas modalidades de atividade física na qualidade de vida sejam realizados para se obter um entendimento claro do efeito dessas intervenções na qualidade de vida desses idosos. ${ }^{30}$

Sugere-se, ainda, que futuros estudos descrevam com mais detalhes as intervenções empregadas, para que elas possam ser replicadas tanto na prática clínica quanto em futuras pesquisas.

\section{CONCLUSÃO}

Os resultados demonstram que há evidência limitada a respeito dos benefícios de exercícios na água e de um programa composto por treino aeróbico associado a exercícios de coordenação e fortalecimento na qualidade de vida de idosos vivendo na comunidade. Em relação ao treino funcional e aos exercícios de fortalecimento muscular, não há evidência a respeito de sua eficácia na qualidade de vida. Entretanto, ainda há poucos estudos a respeito da eficácia da prática 
de atividade física no desfecho qualidade de vida de idosos vivendo na comunidade. Portanto, sugere-se que mais estudos longitudinais com diferentes tipos de intervenções sejam realizados, para que as conclusões a respeito da eficácia dessas intervenções sejam mais robustas.

\section{REFERÊNCIAS}

1. Guccione AA. Fisioterapia geriátrica. Rio de Janeiro: Guanabara Koogan; 2002.

2. Antunes HKM, et al. Alterações cognitivas em idosas decorrentes do exercício físico sistematizado. Rev Bras Med Esporte 2006; 12(2).

3. Naranjo JLR, et al. Autonomía e validismo en la tercera edad. Rev Cubana Med Gen Integr 2001; $17: 22-6$.

4. Paschoal SMP. Qualidade de vida do idoso: construção de um instrumento de avaliação através do método do impacto clínico. [Tese de Doutorado]. São Paulo: USP; 2004.

5. Caromano FA, Ide MR, Kerbauy RR. Exercise maintenance among older adults. Rev Dep Psicol UFF 2006; 18(2): 177-192.

6. Organización Panamericana de la Salud. Lãs condiciones de salud en las Américas. Washington: Organização Panamericana de Saúde; 1994.

7. Instituto Brasileiro de Geografia Estatística. Censo Demográfico de 2000. [acessado em: mar 2006] Disponível em URL: < http:// www.ibge.gov.br >

8. Garrido R, Menezes PR. O Brasil está envelhecendo: boas e más notícias por uma perspectiva epidemiológica. Rev Bras Psiquiatr 2002; 24(1): 3-6.

9. Kalache A, Veras RP, Ramos LR. O Envelhecimento da população mundial: um desafio novo. Rev Saúde Pública 1987; 21(3): 200-10.

10. Pereira RJ, et al. Contribuição dos domínios físico, social, psicológico e ambiental para a qualidade de vida global de idosos. Rev psiquiatr 2006; 28(1): 27-38.

11. The WHOQOL Group. The word health organization quality of life assessment (WHOQOL): position paper from the Word Health Organization. Soc Sc Med 1995.

12. Paúl C, Fonseca AM, Martin I, Amado J. Satisfação e qualidade de vida em idosos portugueses. In: Paul C, Fonseca AM (coords.). Envelhecer em Portugal: psicologia, saúde e prestação de cuidados. Lisboa: Climepsi Editores; 2005; 77-98.
13. Goncalve D, et al. Promoção da qualidade de vida dos idosos portugueses através $d a$ continuidade de tarefas produtivas. Psic, Saúde \& Doenças 2006; 7(1): 137-143.

14. Auquier P, Simeoni MC, Mendizabal H. Approches théoriques et éthodologiques de la qualité de vie liée à la santé. Revue Prevenir 1997; 33: 77-86

15. Kauffman TL, Jackson O. O indivíduo como um todo. In: Kauffman TL. Manual de reabilitação geriátrica. Rio de Janeiro: Guanabara Koogan; 2001. p. 3-33.

16. Minayo MCS, Hartz ZMA, Buss PM. Quality of life and health: a necessary debate. Ciênc Saúde Coletiva 2000; 5(1): 7-18.

17. Sousa L, Galante H, Figueiredo D. Quality of life and well-being of elderly people: an exploratory study in the Portuguese population. Rev Saúde Pública 2003; 37(3): 364-71.

18. Bley D. Nernazza LN. La multiplicité usage du thèrme de qualité de vie. Revue Prevenir 1997; 33: 7-13.

19. Vuori I. Exercise and physical health musculoskeletal health and functional capabilities. Res Q Exerc Sport 1995; 66: 276-85.

20. Caspersen CJ, Powell KE, Christenson GM. Physical activity, exercise, and physical fi tness: defi nitions and distinctions for health-related research. Public Health Rep 1985; 100 (2): 26-31.

21. Shephard RJ. The scientific basis of exercise prescribing for the very old. Journal of the American Geriatrics Society 1990; 38(1): 62-70.

22. Taylor AH. Physical activity and older adults: a review of health benewts and the e Vectiveness of interventions. J Sports Sci 2004; 22: 703-725.

23. Brown DW, et al. Associations between recommended levels of physical activity and health-related quality of life: findings from the 2001 behavioral risk factor surveillance system (BRFSS) survey. Prev Med 2003; 37(5): 520-8.

24. Mota J, et al. Atividade física e qualidade de vida associada à saúde em idosos participantes $\mathrm{e}$ não participantes em programas regulares de atividade física. Rev Bras Educ Fís Esp 2006; 20(3): 219-225. 
25. Lim KC, et al. Aging, health, and physical activity in Korean Americans. Geriatr Nurs 2007; 28(2): 112-9.

26. Van Peppen RP, et al. The impact of physical therapy on functional outcomes after stroke: what's the evidence? Clin Rehabil 2004; 18(8): 833-62.

27. Maher CG, Sherrington C, Herbert RD, Moseley AM, Elkins M. Reliability of the Pedro scale for rating quality ofrandomized controlled trials. Phys Ther. 2003; 83(8): 713-21.

28. Sato D, Kaneda K, Wakabayashi H, Nomura T. The water exercise improves health-related quality of life of frail elderly people at day service facility. Qual Life Res 2007; 16: 15771585.

Recebido: 03/12/2008

Revisado: $27 / 10 / 2009$

Aprovado: 30/11/2009
29. Stiggelbout M, et al. Once a week is not enough: effects of a widely implemented group based exercise programme for older adults. J Epidemiol Community Health 2004; 58: 83-88.

30. De Vreede PL, et al. The effect of functional tasks exercise and resistance exercise on healthrelated quality of life and physical activity. Gerontology 2007; 53(1): 12-20.

31. Yasunaga A, et al. Yearlong physical activity and health-related quality of life in older japanese adults: the nakanojo study. J Aging Phys Act 2006; 14(3): 288-301

32. Sampaio RF, Mancini MC. Estudos de revisão sistemática: um guia para síntese criteriosa da evidência científica. Rev Bras Fisioter 2007; 11(1): 83-89. 
ANEXO 1

Critério de Síntese por Níveis de Evidência

\section{Evidência forte}

Desfecho com resultados estatisticamente significativos em:

- pelo menos dois ECA de alta qualidade, com escore PEDro de pelo menos quatro pontos*.

Evidência moderada

Desfecho com resultados estatisticamente significativos em:

- pelo menos um ECA de alta qualidade $\mathbf{e}$

- pelo menos dois ECAs de baixa qualidade ( $\leq 3$ pontos) ou um ECC de alta qualidade*.

Evidência limitada

Desfecho com resultados estatisticamente significativos em:

- pelo menos um ECA de alta qualidade ou

- pelo menos dois ECC de alta qualidade* (na ausência de ECA de alta qualidade).

Achados indicativos

Desfecho com resultados estatisticamente significativos em:

- um ECC de alta qualidade ou ECA de baixa qualidade* (na ausência de ECAs de alta qualidade) ou

- dois estudos de natureza não experimental com qualidade suficiente* (na ausência de ECAs e ECCs).

Evidência insuficiente ou ausente

- No caso de resultados dos estudos selecionados não atenderem os critérios de nenhum nível acima ou

- no caso de resultados conflitantes (positivamente significativos e negativamente significativos) entre ECAs

e entre ECCs ou

- no caso de ausência de estudos.

* Se o número de estudos que demonstram evidência for inferior a 50\% do número total de estudos dentro de uma mesma categoria de qualidade e desenho metodológico (ECA, ECC, não-experimental) não há classificação de evidência. 